\title{
Construção de um questionário de freqüência alimentar como subsídio para programas de prevenção de doenças crônicas não transmissíveis ${ }^{1}$
}

\section{Development of a food frequency questionnaire as a tool for programs of chronic diseases prevention}

Adriana Bouças RIBEIRO²

Marly Augusto CARDOSO 3

\section{RES U M O}

O presente estudo avaliou a adequação de uma lista de alimentos e a distribuição do tamanho das porções alimentares em versão simplificada de um questionário quantitativo de freqüência alimentar, visando sua adoção em programas de prevenção e controle de doenças crônicas não transmissíveis. Para esta análise, um inquérito recordatório de 24 horas (IR24) foi obtido em amostra aleatória de funcionários da Administração Central da Secretaria de Estado da Saúde de São Paulo $(n=212)$, durante um estudo piloto do programa de combate ao tabagismo. A lista de alimentos do questionário de freqüência alimentar foi avaliada pela contribuição percentual de alguns de seus nutrientes em relação ao consumo total estimado através do IR24. O tamanho das porções alimentares do questionário de freqüência alimentar foi classificado em pequeno, médio e grande de acordo com a distribuição percentual dos pesos correspondentes às medidas caseiras referidas no IR24, utilizando-se os percentis 25,50 e 75, respectivamente.

Termos de indexação: questionário de freqüência alimentar, consumo de alimentos, métodos epidemiológicos, epidemiologia nutricional, inquéritos nutricionais.

\section{A B S T R A C T}

The present study assessed both the food list adequacy and the portion size distribution of each item of the short version of a food frequency questionnaire for programs of chronic diseases prevention. For this analysis,

1 Subvencionado pelo Instituto Nacional de Câncer (INCA) e pela Secretaria de Estado da Saúde de São Paulo.

2 Divisão de Doenças Crônicas Não-Transmissíveis, Centro de Vigilância Epidemiológica, Secretaria de Estado da Saúde de São Paulo. São Paulo, SP, Brasil.

3 Departamento de Nutrição, Faculdade de Saúde Pública, Universidade de São Paulo. Av. Dr. Arnaldo, 715, 01246-904, São Paulo, SP, Brasil. Correspondência para/ Correspondence to: M.A. CARDOSO.E-mail:marlyac@hotmail.com 
a one-day dietary recall (1-DR) was obtained with a sample of 212 health workers randomly selected during a pilot study for a program to combat smoking. The food list evaluation was based on the contribution of some nutrients to the total population intake estimated by the 1-DR. The portion sizes of the food frequency questionnaire items was classified in small, medium and large, according to the distribution of weights equivalent to the measures recorded in the 1-DR (percentiles 25, 50 and 75, respectively).

Index terms: food frequency questionnaire, food consumption, epidemiologic methods, nutritional epidemiology, nutritional surveys.

\section{NTRODUÇÃ O}

O perfil de morbi-mortalidade da população brasileira tem se alterado consideravelmente nas últimas décadas em decorrência do aumento da prevalência de doenças crônicas não transmissíveis (DCNT). Entre estas, destacam-se as doenças cardiovasculares, os cânceres e o diabetes mellitus como as principais causas de morte nos países desenvolvidos e em desenvolvimento (Barreto \& Carmo, 1998).

No Estado de São Paulo, os coeficientes de mortalidade por câncer e diabetes no período de 1980 a 1995 mostraram-se estáveis em ambos os sexos, independentemente da idade. Para as doenças cardiovasculares, embora em declínio, os coeficientes foram bastante elevados, e 30\% destas mortes ocorreram em indivíduos com idade igual ou inferior a 60 anos (São Paulo, 1997).

Fatores de risco relacionados ao estilo de vida, tais como obesidade, tabagismo, sedentarismo, hipercolesterolemia, alcoolismo, estresse e hipertensão arterial, têm sido associados às DCNT (Drewnowski \& Popkin, 1997; Monteiro \& Conde, 1999). A redução da exposição a eles não só contribui para a diminuição da mortalidade, como também pode ser considerada uma variável de impacto para ocorrer um retardo substancial no surgimento destas doenças (Monteiro et al., 1995).

Investigações epidemiológicas sugerem que inúmeros aspectos dietéticos possam estar associados à ocorrência ou prevenção de DCNT. Vários estudos mencionaram o papel protetor do consumo de fibra e vegetais (frutas e hortaliças) na etiologia de doenças crônicas (Steinmetz \& Potter, 1996; Willett, 1998). Tal situação reforça a necessidade de se desenvolver estratégias de intervenção, pois os fatores de risco e de proteção relacionados às DCNT incluem aspectos ambientais. Faz-se necessário o estímulo à mudança de comportamento da população de risco e à divulgação de informações sobre medidas de prevenção e controle destas doenças (Barreto \& Carmo, 1998).

Ações de prevenção primária do câncer no Brasil vêm gradativamente ampliando a sua importância dentro das ações de saúde pública no país. O Ministério da Saúde, através do Instituto Nacional de Câncer (INCA), desenvolve programas educativos voltados para estimular mudanças de comportamento da população, através de três canais comunitários básicos: ambientes de trabalho, escolas e unidades de saúde.

A Coordenação Estadual de Controle do Tabagismo (CETAB) da Secretaria de Estado da Saúde de São Paulo (SES-SP) implantou o programa Secretaria de Estado da Saúde Livre do Cigarro entre funcionários da Administração Central da SES-SP. Este projeto compôs-se das seguintes etapas: 1) levantamento da prevalência de tabagismo através da aplicação de questionário estruturado; 2) intervenções educacionais - palestras, debates, distribuição de material educativo; 3) avaliação a longo prazo do impacto das intervenções na prevalência do tabagismo.

Devido à necessidade de atenção para outros fatores de risco de grande importância, além dos ambientais, o presente estudo foi delineado para desenvolver um Questionário de Freqüência 
Alimentar (QFA) durante um estudo piloto do programa de combate ao tabagismo. O QFA poderá ser adotado em ações de prevenção de DCNT, para avaliação e intervenção nutricionais de indivíduos adultos, vinculadas ou não a outras medidas, como campanhas contra o fumo e a hipertensão, entre outras.

\section{CASUISTICAE MEETODOS}

Uma amostra de funcionários da Administração Central da SES/SP foi selecionada a partir de cadastro de seu Departamento de Recursos Humanos, excluindo-se os empregados em período de férias ou licença durante o estudo. O sorteio da população a ser avaliada foi estratificado por função ou tipo de atividade, segundo sexo e ordem crescente de idade. Os indivíduos escolhidos foram convidados à participação voluntária mediante consentimento oral, assegurando-se a confidencialidade dos dados. Do total de 730 funcionários sorteados para o estudo piloto do programa de combate ao tabagismo da SES/SP, 212 (29\%) responderam um inquérito recordatório de 24 horas utilizado nesta pesquisa.

Uma versão reduzida de QFA com 67 itens alimentares foi elaborada a partir de questionário validado para a comunidade Nipo-Brasileira de São Paulo, excluindo-se os alimentos de origem japonesa (Cardoso \& Stocco, 2000; Cardoso et al., 2001). Foram registradas a freqüência média usual de consumo de cada componente do QFA e a respectiva unidade de tempo (por dia, semana ou mês). O levantamento também incluiu outras questões sobre práticas e preferências alimentares usuais, tais como, ingestão habitual de gordura visível das carnes, tipo de óleo utilizado em saladas e no preparo das refeições, outros alimentos não listados de consumo habitual.

Para identificação das porções alimentares de referência da população de estudo, um inquérito recordatório de 24 horas (IR24) foi obtido entre os participantes da pesquisa. As quantidades de alimentos consumidos foram expressas em medidas caseiras e convertidas em gramas ou mililitros com o auxílio de tabelas nacionais de composição de alimentos (Soares et al., 1992; Pinheiro et al., 1994).

As porções alimentares foram classificadas de acordo com a distribuição percentual dos pesos equivalentes às medidas caseiras referidas no IR24. A porção mediana (M) foi utilizada como referência (percentil 50) em cada item alimentar do QFA, atribuindo-se às quantidades pequena $(P)$ e grande (G) os percentis 25 e 75, respectivamente. Este procedimento permite uma estimativa mais acurada do teor de nutrientes em questionários de freqüência alimentar (Block et al., 1986).

A análise nutricional do IR24 foi realizado com o auxílio do programa WFOOD 2.0, desenvolvido pela Universidade da Califórnia (EUA), que possibilita a análise da composição química dos alimentos, estimando o valor total de diversos nutrientes por refeição ou dia.

Para a avaliação da lista de alimentos e bebidas do QFA foi calculada a contribuição percentual de alguns nutrientes (energia, proteína, gordura total, gordura saturada, colesterol, fibra, vitamina $A$, cálcio, ferro e vitamina $C$ ) em relação ao consumo total estimado através do IR24 para o grupo de indivíduos estudados (Block et al., 1986). Os cálculos foram efetuados com o auxílio do programa de computador Excel.

\section{RESULTADOS}

Dos 212 participantes, 165 (77,8\%) eram mulheres e $47(22,2 \%)$ homens, com idades médias (desvio-padrão) de 43,4 $(10,1)$ e 45,4 $(10,9)$ anos, respectivamente. Os valores médios (desvio-padrão) do Índice de Massa Corporal não diferiram segundo sexo: $24,7(4,9) \mathrm{kg} / \mathrm{m}^{2}$ para mulheres e $24,7(4,2) \mathrm{kg} / \mathrm{m}^{2}$ para homens. Com relação à escolaridade, 86 participantes $(40,6 \%)$ tinham completado curso superior, $68(32,1 \%)$ tinham concluído o segundo grau e $58(27,4 \%)$ 
tinham freqüentado o ensino fundamental, dos quais $22(10,3 \%)$ informaram ter cursado até a $4^{a}$ série.

Para avaliação da dieta habitual da população estudada, o tamanho destas porções foi classificado em pequeno, médio (referência) e grande, de acordo com os pesos correspondentes aos percentis 25, 50 e 75, respectivamente (Anexo).

Para os nutrientes considerados nesta pesquisa, esta contribuição foi superior a $90 \%$ da ingestão total estimada através do IR24 para o grupo de indivíduos estudado. A partir desta análise, foi possível adequar a lista de alimentos do QFA, acrescentando-se alguns itens alimentares de consumo habitual (por exemplo, farofa, sopas, açúcar no café) e retirando-se outros de pequena contribuição nutricional ao consumo total populacional (por exemplo, legumes em conserva) (Tabela 1).

Tabela 1. Contribuição percentual de alguns nutrientes estimados através do Questionário de Freqüência Alimentar em relação ao consumo total obtido em um inquérito recordatório de 24 horas $(n=212)$, Administração Central da Secretaria de Estado daSaúde de São Paulo, 1999

\begin{tabular}{lc}
\hline Nutriente & $\begin{array}{c}\text { Contribuição percentual do } \\
\text { consumo populacional total }\end{array}$ \\
\hline Energia & 94,7 \\
Proteína & 98,7 \\
Gordura total & 97,3 \\
Gordura saturada & 98,4 \\
Fibra da dieta & 96,8 \\
VitaminaC & 98,6 \\
Cálcio & 97,9 \\
Ferro & 96,8 \\
VitaminaA & 97,8 \\
Colesterol & 99,3 \\
\hline
\end{tabular}

\section{DISCUSSÃO}

A elaboração de um instrumento para coleta de dados deve levar em consideração sua finalidade, tempo disponível para sua aplicação e características da população que possam influenciar seu desempenho (escolaridade, práticas habituais, motivação, entre outros aspectos).
Inúmeros Questionários de Freqüência Alimentar (QFA) têm sido elaborados para inquéritos epidemiológicos nacionais e internacionais, visando a avaliação da dieta habitual (Thompson \& Byers, 1994). Estes instrumentos têm se mostrado de fundamental importância não só para investigações sobre a relação entre composição da dieta e saúde (Block et al., 1986), como também para a análise do papel do consumo alimentar na etiologia de doenças crônicas (Willett, 1994).

Apesar de suas desvantagens em relação à limitada exatidão dos dados, devido ao viés de memória do entrevistado e à ausência de informações detalhadas sobre o consumo de alimentos, O QFA possui a capacidade de caracterizar a dieta habitual de cada indivíduo, captar suas mudanças recentes e discriminar a variação de consumo inter-individual, características necessárias à estimativa de riscos. Outra vantagem importante é o seu baixo custo relacionado ao menor intervalo de tempo necessário para o preenchimento (Thompson \& Byers, 1994).

É de grande importância para o desenvolvimento de um QFA o planejamento da lista de alimentos de acordo com as características da dieta habitual e com estimativas das porções alimentares adequadas à ingestão usual da população de estudo, possibilitando assim classificar os indivíduos segundo o padrão de consumo dos nutrientes ingeridos (Pereira \& Koifman, 1999; Cardoso \& Stocco, 2000).

No presente estudo, um questionário previamente validado foi adaptado para ser utilizado em estudos de intervenção e/ou programas de prevenção de doenças crônicas. $\mathrm{O}$ número de itens alimentares foi reduzido de 120 para 67 com o objetivo de simplificar sua aplicação. A lista de alimentos selecionada visou a abrangência das principais fontes alimentares de alguns nutrientes, de acordo com estimativa de consumo do grupo de indivíduos estudado, obtida através de um inquérito recordatório de 24 horas. Assim, para os nutrientes considerados nesta análise, a lista de alimentos desta versão reduzida 
do QFA atingiu mais de $90 \%$ da ingestão total populacional - percentagem adequada para se garantir uma estimativa razoável do consumo de vários outros nutrientes que se correlacionam com a ingestão energética total da dieta (Block et al., 1986). Além da avaliação da lista de alimentos, foram possíveis também a identificação das quantidades correspondentes às porções alimentares referidas pela população estudada e sua classificação em três categorias (pequena, média e grande).

Os procedimentos aqui descritos visando a adaptação da versão simplificada de um QFA para programas de saúde não substituem sua constante avaliação e a validação do instrumento em grupos populacionais com características diferentes dos participantes do presente estudo.

\section{REFERENCIAS BIBLIOGRAFICAS}

BARRETO, M.L., CARMO, E.H. Tendências recentes das doenças crônicas no Brasil. In: Lessa, I. O adulto brasileiro e as doenças da modernidade: epidemiologia das doenças crônicas não-transmissíveis. São Paulo : Hucitec, 1998. p.15-27.

BLOCK, G., HARTMAN, A.M., DRESSER, C.M., CARROLL, M.D., GANNON, J., GARDNER, L. A data-based approach to diet questionnaire design and testing. American Journal of Epidemiology, Baltimore, v.12, n.3, p.453-469, 1986.

CARDOSO, M.A., STOCCO, P.R. Desenvolvimento de um questionário quantitativo de freqüência alimentar em imigrantes japoneses e seus descendentes residentes em São Paulo, Brasil. Cadernos de Saúde Pública, Rio de Janeiro, v.16, n.1, p.107-114, 2000.

CARDOSO, M.A., KIDA, A.A., TOMITA, L.Y., STOCCO, P.R. Reproducibility and validity of a food frequency questionnaire among women of Japanese ancestry living in Brazil. Nutrition Research, New York, v.21, n.5, p.725-733, 2001.

DREWNOWSKI, A., POPKIN, B.M. The nutrition transition: new trends in the global diet. Nutrition Reviews, New York, v.55, n.2, p.31-43, 1997.
MONTEIRO, C.A., MONDINI, L., SOUZA, A.L.M., POPKIN, B.M. Da desnutrição para a obesidade: a transição nutricional no Brasil. In: MONTEIRO, C.A. Velhos e novos males da saúde no Brasil: a evolução do país e de suas doenças. São Paulo : Hucitec, 1995. p.247-255.

MONTEIRO, C.A., CONDE, W.L. A tendência secular da obesidade segundo estratos sociais: Nordeste e Sudeste do Brasil, 1975-1989-1997. Arquivos Brasileiros de Endocrinologia e Metabolismo, São Paulo, v.43, n.3, p.186-194, 1999.

PEREIRA, R.A., KOIFMAN, S. Uso do questionário de freqüência na avaliação do consumo alimentar pregresso. Revista de Saúde Pública, São Paulo, v.33, n.6, p.610-621, 1999.

PINHEIRO, A.B.V., LACERDA, E.M.A., BENZECRY, E.H., GOMES, M.C.S., COSTA, V.M. Tabela para avaliação de consumo alimentar em medidas caseiras. 2.ed. Rio de Janeiro : UFRJ, 1994. 63p.

SÃO PAULO. Secretaria de Estado da Saúde. Centro de Vigilância Epidemiológica Professor Alexandre Vranjac. Divisão de Doenças Crônicas Não Transmissíveis. Indicadores utilizados na vigilância de doenças crônicas não transmissíveis. São Paulo, 1997. 45p.

SOARES, E.A., PORTELLA, E.S., ISHI, M. Relação de medidas caseiras de 320 alimentos e respectivas gramagens. São Paulo : CEANE, 1992. 34p.

STEINMETZ, K.A., POTTER, J.D. Vegetables, fruit and cancer prevention: a review. Journal of the American Dietetic Association, Chicago, v.96, n.10, p.1027-1039, 1996.

THOMPSON, F.E., BYERS, T. Dietary assessment resource manual. Journal of Nutrition, Bethesda, v.124, p.2245S-2317S, 1994. Supplement.

WILLETT, W.C. Future directions in the development of food frequency questionnaires. American Journal of Clinical Nutrition, Bethesda, v.59, p.171S-174S, 1994. Supplement.

WILLETT, W.C. Nutritional Epidemiology. 2.ed. New York : Oxford University Press, 1998. 514p.

Recebido para publicação em 16 de fevereiro e aceito em 29 de junho de 2001. 
244 | A.B. RIBEIRO \& M. A. CARDOSO

\section{A NEXO}

DISTRIBUIÇÃO EM PERCENTIS (P) DO TAMANHO DAS PORÇÕES ALIMENTARES (EM MEDIDAS CASEIRAS E/OU EQUIVALENTES EM GRAMAS OU MILILITROS) REGISTRADAS EM UM INQUÉRITO RECORDATÓRIO DE 24 HORAS $(N=212)$, ADMINISTRAÇÃO CENTRAL DA SECRETARIA DE ESTADO DA SAÚDE DE SÃO PAULO, 1999

\begin{tabular}{|c|c|c|c|}
\hline Alimentos e Preparações & P50 & P25 & P75 \\
\hline Abacate & 1 xicara das de chá $(130 \mathrm{~g})$ & $1 / 4$ unidade $(80 \mathrm{~g})$ & $1 / 2$ unidade $(215 \mathrm{~g})$ \\
\hline Alface ou escarola & 3 folhas (30 g) & 2 folhas $(20 \mathrm{~g})$ & 4 folhas $(40 \mathrm{~g}$ ) \\
\hline Amendoim & 1⁄2xícara das de chá $(50 \mathrm{~g})$ & $25 \mathrm{~g}$ & $100 \mathrm{~g}$ \\
\hline Arrozbranco & 1 escumadeira $(77,5 \mathrm{~g})$ & 3 colheres das de sopa (60 g) & 2 escumadeiras (145 g) \\
\hline Aveia/granola & 3 colheres das de sopa ( $26 \mathrm{~g}$ ) & $15 \mathrm{~g}$ & $50 \mathrm{~g}$ \\
\hline Banana & 1 unidade (60 g) & 1 unidade pequena $(50 \mathrm{~g})$ & 2 unidades $(120 \mathrm{~g})$ \\
\hline Batata-doce/abóbora & 1 unidade $(70 \mathrm{~g})$ & $50 \mathrm{~g}$ & 4 colheres das de sopa $(120 \mathrm{~g})$ \\
\hline Batata frita & 2 colheres das de sopa ( $50 \mathrm{~g}$ ) & $1 / 2$ pegador $/ 1$ colher das de sopa $(25 \mathrm{~g})$ & 1 porção (100 g) \\
\hline Batata/mandioca & 1 unidade $(70 \mathrm{~g})$ & $50 \mathrm{~g}$ & 2 unidades $(140 \mathrm{~g})$ \\
\hline Berinjela & 2 colheres das de sopa ( $50 \mathrm{~g}$ ) & $30 \mathrm{~g}$ & 3 colheres das de sopa ( $80 \mathrm{~g})$ \\
\hline Beterraba & 3 colheres das de sopa ( $50 \mathrm{~g}$ ) & $25 \mathrm{~g}$ & 1 unidade $(80 \mathrm{~g})$ \\
\hline Biscoito/torrada & 3 unidades $(21 \mathrm{~g})$ & 2 unidades $(14 \mathrm{~g})$ & 4 unidades (28g) \\
\hline Bolo/tortas/pavês & 1 fatia/1 pedaço (100 g) & $50 \mathrm{~g}$ & $11 \frac{1}{2}$ pedaço $(150 \mathrm{~g})$ \\
\hline Café & $1 / 2$ copo pequeno $(75 \mathrm{~mL})$ & 1 xícara $(50$ mL) & 2 xícaras $(100 \mathrm{~mL})$ \\
\hline Camarão/ula & 1 escumadeira (120 g) & $50 \mathrm{~g}$ & $150 \mathrm{~g}$ \\
\hline Carnebovina & 1 unidade média (100 g) & $75 \mathrm{~g}$ & 1 unidade grande $(120 \mathrm{~g})$ \\
\hline Carne defrango & 1 filé (100 g) & $65 \mathrm{~g}$ & 3 pedaços (135g) \\
\hline Carne de porco & 1 unidade (165 g) & $77,5 \mathrm{~g}$ & $255 \mathrm{~g}$ \\
\hline Cenoura & 2 colheres das de sopa ( $25 \mathrm{~g}$ ) & 1 colher das de sopa $(12 \mathrm{~g})$ & 4 colheres das de sopa (48 g) \\
\hline Cenveja & 350 mL (1 lata) & 300 mL(1 copo grande) & $600 \mathrm{~mL}$ (1 garrafa) \\
\hline Chádeenvas & 1 xícara $(200 \mathrm{~mL})$ & $150 \mathrm{~mL}$ & $300 \mathrm{~mL}$ \\
\hline Chá preto & 1 xícara $(200 \mathrm{~mL})$ & $150 \mathrm{~mL}$ & $300 \mathrm{~mL}$ \\
\hline Chocolates & 2 unidades/1 barra (30 g) & 1 unidade (15g) & 5 unidades (75 g) \\
\hline Couve/brócolos & 3 colheres das de sopa ( $45 \mathrm{~g}$ ) & $25 \mathrm{~g}$ & $80 \mathrm{~g}$ \\
\hline Doce de abóbora & 1 colher das de sopa $(30 \mathrm{~g})$ & $20 \mathrm{~g}$ & $50 \mathrm{~g}$ \\
\hline Embutidos & 1 unidade $(60 \mathrm{~g})$ & 4 fatias (40 g) & 2 unidades $(100 \mathrm{~g})$ \\
\hline Envilha/lentilha & $11 / 2$ colher das de sopa $(30 \mathrm{~g})$ & 1 colher das de sopa $(20 \mathrm{~g})$ & 3 colheres das de sopa ( $60 \mathrm{~g}$ ) \\
\hline Farofa & 2 colheres das de sopa ( $25 \mathrm{~g}$ ) & 3 colheres das de chá $(15 \mathrm{~g})$ & $30 \mathrm{~g}$ \\
\hline Feijão roxo & 1 concha (110g) & $1 / 2$ concha $(55 g)$ & 1 concha cheia (140 g) \\
\hline Feijoada & 1 concha cheia $(225 \mathrm{~g})$ & $150 \mathrm{~g}$ & - \\
\hline Geléia & 1 colher das de sopa $(15 \mathrm{~g})$ & $1 / 2$ colher das de sobremesa $(9 \mathrm{~g})$ & 1 colher das de sobremesa $(18 \mathrm{~g})$ \\
\hline logurte & 1 copo (200 mL) & $150 \mathrm{~mL}$ & $250 \mathrm{~mL}$ \\
\hline Laranja & 1 unidade (180 g) & $125 \mathrm{~g}$ & 2 unidades (360 g) \\
\hline Leite com café & 1 copo pequeno $(150 \mathrm{~mL})$ & $100 \mathrm{~mL}$ & 1 copo (250 mL) \\
\hline Leite desnatado & 1 copo pequeno $(150 \mathrm{~mL})$ & $100 \mathrm{~mL}$ & 1 copo (250 mL) \\
\hline Leitesem café & 1 copo pequeno (150 mL) & $100 \mathrm{~mL}$ & 1 copo (250 mL) \\
\hline Maçã & 1 unidade pequena $(80 \mathrm{~g})$ & $60 \mathrm{~g}$ & 1 unidade grande $(130 \mathrm{~g})$ \\
\hline Maionese & 1 colher das de sopa $(15 \mathrm{~g})$ & 2 colheres das de chá $(7 \mathrm{~g})$ & 2 colheres das de sopa ( $30 \mathrm{~g})$ \\
\hline Mamão & $1 / 2$ unidade $(155 \mathrm{~g})$ & 1 fatia fina (100 g) & 1 fatia média (170 g) \\
\hline Manga/caqui & 4 pedaços (100 g) & $45 \mathrm{~g}$ & 1 unidade $(180 \mathrm{~g})$ \\
\hline Manteiga & 2 pontas de faca $(5 \mathrm{~g})$ & 1 colher das de café $(3 \mathrm{~g})$ & $7 \mathrm{~g}$ \\
\hline Margarina & 1 ponta de faca $(2,5 \mathrm{~g})$ & 1 colher das de café $(3 \mathrm{~g})$ & 2 colheres das de café $(6 \mathrm{~g})$ \\
\hline
\end{tabular}




\begin{tabular}{|c|c|c|c|}
\hline Massas & 1 prato sobremesa $(95 \mathrm{~g})$ & 1 escumadeira (45g) & 1 prato $(200 \mathrm{~g})$ \\
\hline Melancia/melão & 1 fatia média (90 g) & $55 \mathrm{~g}$ & $150 \mathrm{~g}$ \\
\hline Miúdos & 2 pedaços $(100 \mathrm{~g})$ & $65 \mathrm{~g}$ & $130 \mathrm{~g}$ \\
\hline Outras frutas & 1 unidade $(60 \mathrm{~g})$ & $1 / 2$ unidade $(30 \mathrm{~g})$ & $75 \mathrm{~g}$ \\
\hline Ovo & 1 unidade (50 g) & $1 / 2$ unidade $(25 \mathrm{~g})$ & 2 unidades (100 g) \\
\hline Pão francês/forma & 1 unidade (50 g) & $1 / 2$ unidade $(25 \mathrm{~g})$ & $11 / 2$ unidade $(75 \mathrm{~g})$ \\
\hline Pão integral/trigo & 2 fatias (50 g) & 1 fatia ( $25 \mathrm{~g})$ & 3 fatias $(75 \mathrm{~g})$ \\
\hline Pão sovado/doce & 2 bisnaguinhas ( $40 \mathrm{~g}$ ) & $25 \mathrm{~g}$ & $50 \mathrm{~g}$ \\
\hline Pastel salgado & 1 unidade grande $(110 \mathrm{~g})$ & 1 unidade $(70 \mathrm{~g})$ & $150 \mathrm{~g}$ \\
\hline Peixe & 1 filé (130 g) & $100 \mathrm{~g}$ & $230 \mathrm{~g}$ \\
\hline Pinga/uísque/vodca & $68 \mathrm{~mL}\left(1 \frac{1}{2}\right.$ dose) & $45 \mathrm{~mL}$ (1 dose) & $90 \mathrm{~mL}$ (2 doses) \\
\hline Pizza & $11 \frac{1}{2}$ fatia $(180 \mathrm{~g})$ & $1 / 2$ fatia $(50 \mathrm{~g})$ & 3 fatias (300 g) \\
\hline Presunto/mortadela & $11 \frac{1}{2}$ fatia $(22 \mathrm{~g})$ & 1 fatia (15g) & 2 fatias (30 g) \\
\hline Pudins/flans & 1 unidade (100 g) & 1 unidade pequena $(50 \mathrm{~g})$ & 2 pedaços (150 g) \\
\hline Queijo amarelo & 1 fatia (20 g) & 1 fatia fina $(15 \mathrm{~g})$ & 2 fatias (30 g) \\
\hline Queijo fresco/ricota & 1 fatia (30 g) & 1 fatia fina $(20 \mathrm{~g})$ & 1 fatia grossa $(40 \mathrm{~g})$ \\
\hline Refrigerante diet & 1 copo grande $(300 \mathrm{~mL})$ & 1 copo americano ( $200 \mathrm{~mL})$ & $350 \mathrm{~mL}$ \\
\hline Refrigerantenormal & 1 copo grande ( $300 \mathrm{~mL})$ & 1 copo americano $(200 \mathrm{~mL})$ & $350 \mathrm{~mL}$ \\
\hline Repolho, acelga & 2 a 3 colheres das de sopa ( $45 \mathrm{~g}$ ) & 2 colheres das de sopa ( $30 \mathrm{~g}$ ) & 5 colheres das de sopa $(75 \mathrm{~g})$ \\
\hline Requeijão & 1 colher das de sobremesa $(20 \mathrm{~g})$ & $1 / 2$ colher das de sobremesa $(10 \mathrm{~g})$ & 2 colheres das de sobremesa ( $40 \mathrm{~g}$ ) \\
\hline Salgadinhos & $50 \mathrm{~g}$ & $40 \mathrm{~g}$ & $150 \mathrm{~g}$ \\
\hline Sardinha, atum & $105 \mathrm{~g}$ & $62,5 \mathrm{~g}$ & $180 \mathrm{~g}$ \\
\hline Sopas & 1 prato fundo $(520 \mathrm{~g})$ & $1 / 2$ prato $(290 \mathrm{~g})$ & $1 \frac{1}{2}$ prato $(780 \mathrm{~g})$ \\
\hline Sorvetes & 1 unidade (80 g) & $70 \mathrm{~g}$ & 2 unidades (160 g) \\
\hline Suco laranja natural & 1 copo americano (200 mL) & 1 copo pequeno (165 mL) & 1 copo requeijão ( 250 mL) \\
\hline Suco outras frutas & 1 copo grande $(300 \mathrm{~mL})$ & 1 copo americano $(200 \mathrm{~mL})$ & $2 \operatorname{copos}(600 \mathrm{~mL})$ \\
\hline Sucos artificiais & 1 copo grande (300 mL) & 1 copo americano $(200 \mathrm{~mL})$ & $2 \operatorname{copos}(600 \mathrm{~mL})$ \\
\hline Tomatecru & 1 unidade pequena $(50 \mathrm{~g})$ & 3 rodelas $(30 \mathrm{~g})$ & $80 \mathrm{~g}$ \\
\hline Uva & 1 cacho pequeno $(75 \mathrm{~g})$ & $40 \mathrm{~g}$ & $150 \mathrm{~g}$ \\
\hline Vagem/chuchu & 2 colheres das de sopa ( $40 \mathrm{~g}$ ) & 1 colher das de sopa $(20 \mathrm{~g})$ & $65 \mathrm{~g}$ \\
\hline Vegetais mistos & 1 pires $(90 \mathrm{~g})$ & $65 \mathrm{~g}$ & $1 / 2$ prato $(115 \mathrm{~g})$ \\
\hline Vinho & 2 cálices $(100 \mathrm{~mL})$ & $60 \mathrm{~mL}$ & $200 \mathrm{~mL}$ \\
\hline
\end{tabular}

